\title{
Using Mobile Agent Results to Create Hard-to-Detect Computer Viruses
}

\author{
Yongge Wang \\ Certicom Corp. \\ 5520 Explorer Drive 4 th Floor \\ Mississauga, Ontario, L4W 5L1, Canada \\ and \\ Center for Applied Cryptographic Research \\ University of Waterloo, ON, N2L $3 G 1$ \\ ygwang@cacr.math.uwaterloo.ca
}

\begin{abstract}
The theory of computer viruses has been studied by several authors, though there is no systematic theoretical study up to now. The long time open question in this area is as follows: Is it possible to design a signature-free (including dynamic signatures which we will define late) virus? In this paper, we give an affirmative answer to this question from a theoretical viewpoint. We will introduce a new stronger concept: dynamic signatures of viruses, and present a method to design viruses which are static signature-free and whose dynamic signatures are hard to determine unless some cryptographic assumption fails. We should remark that our results are only for theoretical interest and may be resource intensive in practice.
\end{abstract}

\section{INTRODUCTION}

The term computer virus is often used to indicate any software that can cause harm to systems or networks. People often does not include certain malicious software, such as Trojan horses and network worms, into the computer virus family. In our discussion, a computer virus refers to any code that cause computer or network systems to behave in a different manner than the desired one.

A Trojan horse program is a useful or apparently useful program or a shell script containing hidden code that performs some unwanted func-

The original version of this chapter was revised: The copyright line was incorrect. This has been corrected. The Erratum to this chapter is available at DOI: 10.1007/978-0-387-35515-3_53 
tion. A simple example of a Trojan horse program might be a telnet program. When a user invokes the program, it appears to be performing telneting and nothing more, however it may also be quietly changing the file access permissions. Some Trojan horse programs are difficult to detect, for example, a compiler on a multi-user system that has been modified to insert additional code into certain programs when they are compiled (see Thompson [8]).

The hidden code of a Trojan horse program is deliberately placed by the program's author. Generally, the hidden code in a computer virus program is added by another program, that program itself is a computer virus. Thus one of the typical characteristics of a computer virus is to copy its hidden code to other programs, thereby infecting them.

Generally, a computer virus exhibits three characteristics (see, e.g., [9]): a replication mechanism, an activation mechanism, and an objective. The replication mechanism performs the following functions: search for other programs to infect, when finds a program, possibly determines whether the program has been previously infected, insert the hidden instructions somewhere in the program, modifies the execution sequence of the program's instruction such that the hidden code will be executed whenever the program is invoked, and set some flags indicating that the program has been infected. The flag may be necessary because without it, programs could be repeatedly infected and grow noticeably large.

The activation mechanism checks for the occurrence of some event. When the event occurs, the computer virus executes its objective, which is generally some unwanted, harmful action.

Anti-virus tools performs three basic functions: detect, identify, or remove viruses. Detection tools perform proactive detection, active detection, or reactive detection. That is, they detect a virus before it executes, during execution, or after execution. Identification and removal tools are more straightforward in their application.

For detection of viruses, there are five classes of techniques (see, e.g., [5]): signature scanning and algorithmic detection, general purpose monitors, access control shells, checksums for change detection, and heuristic binary analysis. Note that after the paper [5], a new technique emulation detection has been extensively used to detect polymorphic viruses. In this paper, we will concentrate on designing viruses which are signaturefree and we will not discuss the techniques for identification and removal.

A common class of anti-virus tools employs the complementary techniques of signature scanning and algorithmic detection. This class of tools are known as scanners. Scanners are limited intrinsically to the detection of known viruses. In signature scanning an executable is searched for a selected binary code sequence, called a virus signature. 
General purpose monitors protect a system from the replication of viruses by actively intercepting malicious actions. Access control shells function as part of the operating system, much like monitoring tools. Rather than monitoring for virus-like behavior, the shell attempts to enforce an access control policy for the system. Change detection works on the theory that executables are static objects; therefore, modification of an executable implies a possible virus infection. However, this theory has a basic flaw: some executables are self-modifying. Heuristic binary analysis is a method whereby the analyzer traces through an executable looking for suspicious, virus-like behavior. If a program appears to perform virus-like actions, a warning is displayed.

Indeed, the signature scanner is the mostly used technique to detect viruses. Note that at the beginning of this section we mentioned that at the end of the replication mechanism, the virus will generally put a flag in the infected program (this flag can often be included in the signature of the virus). Otherwise the program may be repeatedly infected, and the size may increase geometrically. Hence the virus will be detected easily.

The authors of computer viruses always try to design viruses that are immune from those anti-virus software on market. A polymorphic virus (see, e.g., $[5,7]$ ) creates copies during replication that are functionally equivalent but have distinctly different byte streams. To achieve this, the virus may randomly insert superfluous instructions, interchange the order of independent instructions, or choose from a number of different encryption schemes. This variable quality makes the virus difficult to locate, identify, and remove. This kind of virus can be thought as static signature-free viruses. However, up to my knowledge, all known polymorphic viruses can be detected by algorithmic detection (or emulation detection) due to their dynamic signatures.

In this paper, we will introduce a new concept: dynamic signatures of viruses and present a method to design viruses which are static signaturefree and whose dynamic signatures are hard to determine unless some cryptographic assumption fails. This will answer a long time open question in this area.

\section{NOTATIONS}

Turing machines are the basic model for computation. We will present our construction in terms of Turing machines. Our notations are standard, e.g., as those in Hopcroft and Ullman [3]. We will use two-way infinite tape, multi-track, and multi-tape Turing machines as our model of computation. Formally, a Turing machine (TM) is denoted $M=(Q$, 
$\left.\Sigma, \Gamma, \delta, q_{0}, B, F\right)$ where $Q$ is the finite set of states, $\Gamma$ is the finite set of allowable tape symbols, $B$ is the blank symbol which belongs to $\Gamma, \Sigma$ is the set of input symbols which is a subset of $\Gamma$ not including $B, \delta$ is the next move function which is a mapping from $Q \times \Gamma$ to $Q \times \Gamma \times\{L, R\}$ ( $\delta$ may, however, be undefined for some arguments), $q_{0} \in Q$ is the start state, and $F \subseteq Q$ is the set of final states. For each TM $M$, there is a read only input tape and a write only output tape.

Without loss of generality, we will assume that $\Sigma=\{0,1\}$ in this paper unless specified otherwise. The set of strings over $\Sigma$ will be denoted as $\Sigma^{*}$, and the set of length $n$ strings will be denoted as $\Sigma^{n}$. A subset of $\Sigma^{*}$ is called a set, a problem, or just a language. The characteristic function of a set $A$ is defined by letting $A(x)=1$ if $x \in A$ and $A(x)=0$ otherwise.

\section{GENERATING UNDETECTABLE SIGNATURES}

From our analysis in previous sections, in order for a virus to be undetectable, the virus must have some mechanism to check whether a program has already been infected by it or not. For this purpose, most viruses will put some flags in infected programs. These flags generally can be considered as signatures. Formally, the signature of a virus is a byte sequence by which the virus can be distinguished from other programs or viruses. That is, the virus code or programs infected by it contain this byte sequence, but all other programs or viruses generally do not contain this byte sequence. This definition of virus signature is a static one (which is generally used in the literature). In this paper, we will introduce a new concept: a virus can have a dynamic signature. Formally, a dynamic signature of a virus is a function which can be used to distinguish it from other programs or viruses. From the definition, it is clear that dynamic signatures of viruses can only be determined by simulating the execution of the virus or the programs infected by it.

It is clear that each non-trivial virus should have either a static signature or a dynamic signature. Polymorphic viruses do not have static signatures, but generally they have dynamic signatures. The reason why all previous polymorphic viruses can be detected by current anti-virus software in market is that their dynamic signatures are generally easy to determine.

Obviously, viruses which have static signatures or whose dynamic signatures are easy to determine can easily be detected. In order for a virus to be undetectable, it should have the following properties:

- they have no static signatures in infected programs, 
- their dynamic signatures are undetectable, for example, their dynamic signatures are hard to detect unless some cryptographic assumption fails.

In this section, we present a method to design undetectable dynamic signatures. In the next section, we will present a method for a virus to infect programs so that it leaves no static signature and in the same time keeps the dynamic signature undetectable.

Let $F: N \rightarrow N$ be a secure function with the following properties:

- $|F(x)|=|x|$ for all $x \in \Sigma^{*}$,

- Given the values of $F$ on some given set $A \subset N$, it is hard for the enemy to compute any value of $F$ on $x \notin A$,

where $N$ is the set of positive integers. Such kind of functions can be chosen from any variant of digital signature schemes or some encryption schemes, for example, a possible candidate is the encryption scheme of Cramer and Shoup which is secure against adaptive chosen ciphertext attack. The reader is referred to [4] for more details on digital signature schemes and encryption schemes..

A set $D$ is called super sparse if $\left|D \cap \Sigma^{n}\right| \leq 1$ for all $n$ and $|D|=\infty$. For each virus we will assign a super sparse set $D$ as its dynamic signature. Note that only the virus creator knows the dynamic signature of the virus. It should also be the case that it is computationally hard to guess the dynamic signature of any virus. Let $n_{0}>100$ be a large enough integer and $F$ be a secure function as mentioned above. Then the dynamic signature for a virus is assigned as follows:

$$
D_{v}=\left\{x \in \Sigma^{n}: x \text { is the binary representation of } F(n), n>n_{0}\right\}
$$

Our method to insert the dynamic signature into a virus is as follows: the virus will modify the behavior of the host program such that the program will output random values of 0 or 1 on inputs from $D_{v}$. Here we assume that the host program is a Turing machine $M$ which computes the characteristic function of a set $A$ (that is, $A$ means the normal behavior of the program). Then the infected program will be a Turing machine $M^{\prime}$ with the properties that: $M^{\prime}(x)=A(x)$ for all $x \notin D_{v}$ and $M^{\prime}(x)$ is a randomly chosen element from $\Sigma(=\{0,1\})$ for $x \in D_{v}$. From the property of randomness, $M^{\prime}(x)=1$ for approximately one half inputs $x$ from $D_{v}$.

Note that if $n_{0}$ is large enough, then the set $D_{v}$ is a super sparse subset of $\Sigma^{*}$, whence the terminal user will not notice the existence of the virus. Also any anti-virus program will not be able to detect the virus unless one of the following conditions holds: 
1. the anti-virus program can search a space of at least $2^{2 n_{0}}$;

2. the function $F$ can be broken, that is, the anti-virus program can compute the value $F(n)$ for sufficiently many $n>n_{0}$. (Indeed, in practice, the anti-virus program even does not know the function $F)$.

Let $M_{D_{v}}$ be the Turing machine that models a virus with a dynamic signature $D_{v}$. That is, $M_{D_{v}}$ has the following properties: $1 . M_{D_{v}}$ computes the desired virus function (or behavior); $2 . M_{D_{v}}$ outputs randomly chosen elements from $\Sigma$ for inputs from $D_{v}$.

\section{CHECKING INFECTED PROGRAMS}

Each time when a virus find a chance to infect a program, it will first decide whether it has already infected the target program or not. For a virus with a static signature, this will be straightforward. For a virus with a dynamic signature (but without static signature), this can be done by virtually simulating the execution of the target program on some inputs. The following algorithm can be used for this purpose.

\section{Checking algorithm:}

1. Let $c=0, j=n_{0}+1$, and $K_{0}$ be a specific reasonably large integer (e.g., $\left.K_{0}=100000\right)$;

2. Let $x_{j}$ be the binary representation of $F(j)$. We distinguish the following two cases:

Case 1: $M\left(x_{j}\right)=1$. Let $c=c+1$ and $j=j+1$.

Case 2: $M\left(x_{j}\right)=0$. Let $j=j+1$.

3. If $j<K_{0}$ then go to (2) else go to (4).

4. If $c \approx \frac{K_{0}-n_{0}}{2}$ then $M$ has been infected. Otherwise, $M$ is not infected.

Note that the above checking algorithm sometime may output wrong information. That is, sometime it will consider a non-infected program as infected. However, this will not be a big problem for a virus. The purpose of a virus is to infect many programs, and does not need to infect all programs. Also it should be noted that if one can observe one execution of the above checking algorithm, then one can easily determine an initial part of the dynamic signature $D_{v}$, whence help the anti-virus 
software to detect the virus. In order to solve this problem, we can use the protocol of computation with encryption (see [1]).

Abadi and Feigenbaum [1] described a protocol how to securely evaluate a Boolean circuit in encrypted data. They further reduced the problem of evaluation of encrypted functions to the problem of processing of encrypted data by representing the Boolean circuit that is to be hidden as data fed to a universal Boolean circuit. Sander and Tschudin ([6]) have considered the similar problems in the context of protecting mobile agents against malicious hosts. They have shown that it is possible for a mobile agent to actively protect itself against its execution environment that tries to get some information of the agent for some malicious goal. Especially, they have identified a special class of functions - polynomials and rational functions - together with encryption schemes that lead to a non-trivial example of cryptographically hiding a function such that it can nevertheless be executed with a non-interactive protocol. That is, mobile agents executing this class of functions can protect themselves against tampering by a malicious host and can conceal the programs they want to have executed. Obviously our above checking algorithm can be considered as a Boolean function of Abadi and Feigenbaum [1], though generally it does not belong to the function classes identified by Sander and Tschudin ([6]). In practice, a virus writer may find some trade-off between the results of Abadi and Feigenbaum [1] and Sander and Tschudin [6], and achieve the following goal: it can check whether a program has already been infected but not leak any information of its dynamic signature.

From the discussion of this section, we can model a virus with a dynamic signature $D_{v}$ as a Turing machine $M_{D_{v}}$ with the following properties: 1. $M_{D_{v}}$ computes the desired virus function (or behavior); 2. $M_{D_{v}}$ outputs randomly chosen elements from $\Sigma$ for inputs from $D_{v} ; 3 . M_{D_{v}}$ can check whether a given program has already been infected without leaking any information about its dynamic signature $D_{v}$.

In the next section, we will give a method to embed this virus $M_{D_{v}}$ into any host program without leaving any static signature.

\section{INSERTING A VIRUS INTO A PROGRAM}

Assume that we have a Turing machine $M$ (that is, a program) which computes the characteristic function of a set $A$. That is, for each $x \in \Sigma^{*}$, $M(x)=A(x)$. In order to infect $M$ with a virus $M_{D_{v}}$, we will combine the two Turing machines into one Turing machine and introduce randomness into the codes so that no static signature is left in the combined 
Turing machine. For the reason of simplicity, we will assume that the virus Turing machine $M_{D_{v}}$ will only compute the following function

$$
M_{D_{v}}(x)= \begin{cases}? & x \notin D_{v} \\ 0 \text { or } 1 & x \in D_{v}\end{cases}
$$

Of course, a virus will do many other harmful things in practice. Our results can be easily extended to these harmful viruses since we can regard the host program Turing machine $M$ and the harmful part $M_{v}$ of the virus as one single Turing machine $\bar{M}$ and then apply our method (note that it is trivial to combine two Turing machines into one single Turing machine). Now the following problem remains to be addressed: For each virus Turing machine $M_{D_{v}}$ and a host program Turing machine $M$, how to construct a Turing machine $M^{\prime}$ which computes the function

$$
\operatorname{vcomb}\left\{A(x), M_{D_{v}}(x)\right\}= \begin{cases}A(x) & \text { if } M_{D_{v}}(x)=? \\ M_{D_{v}}(x) & \text { otherwise }\end{cases}
$$

such that from $M^{\prime}$ it is computationally hard to get any information of $D_{v}$ ? That is, how to construct $M^{\prime}$ such that the anti-virus software cannot construct sufficient many elements of $D_{v}$ ? Note that the antivirus software may have no time to run the $M^{\prime}$ s on all inputs to get some information of the dynamic signature $D_{v}$.

\section{One way vcomb-combination of Turing machines}

A Turing machine $M$ is called a vcomb-combination of TMs $M_{1}$ and $M_{2}$ if $M(x)=\operatorname{vcomb}\left\{M_{1}(x), M_{2}(x)\right\}$ for all $x \in \Sigma^{*}$. A Turing machine $M$ is called a composition of TMs $M_{1}$ and $M_{2}$ if $M(x)=M_{2}\left(M_{1}(x)\right)$ for all $x \in \Sigma^{*}$. It is widely believed that in practice it is hard to decompose a Turing machine into two parts. However, if $M$ is just a simple composition of two TMs $M_{1}$ and $M_{2}$, then obviously one can construct $M_{1}$ (and $M_{2}$ ) from $M$ easily.

We can present a method for constructing the vcomb-combination $M$ of two TMs $M_{1}$ and $M_{2}$ with the properties that it is hard for the adversary to construct a TM $M_{2}^{\prime}$ which is almost equivalent to $M_{2}$ in semantics, that is, $M_{2}^{\prime}(x)=M_{2}(x)$ for sufficiently many (the exact number depends on applications) $x \in \Sigma^{*}$. Due to the space limit, the construction details are omitted here. The interested readers are referred to the final version of this paper. Our construction will imply the following theorems.

Theorem 5..1 Let $f$ be an algorithm to construct $M_{2}$ from $M$, then $f$ can be used to compute the permutation $R$. 
Corollary 5..2 The probability that one can decompose $M$ into $M_{1}$ and $M_{2}$ equals to $2^{2 k+8}$ !.

Theorem 5..3 There is an efficient process to construct from any two given Turing machines $M_{1}$ and $M_{2}$ a vcomb-combination Turing machine $M$ with the following properties: given $M$ and $M_{1}$, with extremely high probability, one cannot get any information of $M_{2}$.

\section{Signature undetectability}

Due to the construction process, it is straightforward that our virus will not have any static signature. The dynamic signature of our virus is also hard to detect. First, by Corollary $5 . .2$ and Theorem 5..3, with extremely high probability the anti-virus software cannot get a description of Turing machine $M_{D_{v}}$ for the dynamic signature. Whence, from a static analysis of the infected program, the anti-virus software cannot get any information of the dynamic signature of the virus. Secondly, when the anti-virus software simulates a virtual execution of the virus (or the infected program), it can monitor the process that the virus checks whether a program has already been infected and the process that the virus inserts the hidden virus code into a program. However, as mentioned in section 4., special techniques can be used to avoid the leakage of the dynamic sugnature.

\section{CONCLUSIONS}

Even though our results show that a virus could be written in such a way that its dynamic signature is hard to detect, each virus is still detectable. For example, when we notice the existence of a virus, we can write a simple program and let it be infected. Then we can modify this infected program as the virus detector as follows: run this program in a protected environment and activate its virus infection code. If this program decided not to infect the target code, then with high probability that the taget code is already infected by this virus. This implies that theoretically all known viruses can be detected. However this method is infeasible in practice. The main difficulty here is that there may be millions of different viruses. If we include all of these viruses into the anti-virus software package, the package will have a huge size, and the detecting process will be extremely slow.

In this paper, we have used some results from mobile agents to improve the quality of viruses. Indeed, there is a close relationship between viruses and mobile agents. In some sense, network worms could be considered as the prototype of mobile agents. Mobile agents have got extreme attention recently. We are sure that any future breakthrough 
in mobile agents protection will also be a breakthrough for the design of undetectable viruses.

As have been noticed by computer virus research community, the best way to protect computer and network systems against viruses is to use digital signatures. That is, each time when a computer application package is developed, a digital signature of that software should also be available to the users. This will also defeat the viruses we have designed in this paper. However, this is difficult to achieve in practice. People like to download some shareware (e.g., games) from Internet and run it. For this kind of software, you have to trust the author. Even if you trust the share ware author, it is practically difficult to include the signature keys of all shareware writers in the virus scanner. Hence the virus may be written in such a way that it will only infect this kind of shareware.

\section{References}

[1] M. Abadi and J. Feigenbaum. Secure circuit evaluation. Journal of Cryptology, 2(1):1-12, 1990.

[2] P. Denning. Computer viruses. American Scientist, Vol 76, 1988.

[3] J. Hopcroft and J. Ullman. Introduction to Automata Theory, Languages, and Computation. Addison-Wesley, 1979.

[4] A. Menezes, P. van Oorschot, and S. Vanstone. Handbook of Applied Cryptography. CRC Press, 1996.

[5] W. Polk and L.Bassham. A guide to the selection of anti-virus techniques. National Institute of Standards and Technology, Computer Security Division, 1992.

[6] T. Sander and C. Tschudin. Towards mobile cryptography. In:Proc. of the 1998 IEEE Symposium on Security and Privacy, 1998.

[7] F. Skulason. The mutation engine - the final nail? Virus Bulletin, pp. 11-12, April, 1992.

[8] K. Thompson. Reflections on trusting trust. Commun. ACM, 27(8):761-763, 1984.

[9] J. Wack and L. Carnahan. Computer viruses and related threats: a management guide. NIST Special Publication 500-166, 1989. 